\title{
Three-dimensional Analysis of Non-stained Polymer Alloy Using Differential Phase Contrast-STEM Tomography
}

\author{
Shin Inamoto ${ }^{1 *}$, Akiyo Yoshida ${ }^{1}$ and Yuji Otsuka ${ }^{1}$ \\ 1. Toray Research Center, Inc., Otsu, Shiga, Japan \\ * Corresponding author: shin_inamoto@trc.toray.co.jp
}

Scattering contrast of polymer materials is quite low, since polymer materials are composed mostly of light elements. In order to enhance the scattering contrast, electron staining with metal oxide such as $\mathrm{OsO}_{4}$ and $\mathrm{RuO}_{4}$ is generally used for TEM observations of polymer materials [1]. However, the molecular structures of the polymer materials are destroyed in the staining process due to such as bond dissociations and cross-linking effects. Therefore, observation methods for non-stained polymer materials are strongly required.

Differential phase contrast scanning transmission electron microscopy (DPC-STEM) has been developed to analyze electrical or magnetical fields inside materials [2, 3]. If electrical field derived from the difference of electrostatic potentials is detectable by DPC-STEM, positions of the interface between the different polymer materials are determined. Moreover, electrical field distributions are transformed to phase contrast images through the two-dimensional integration process [4]. The phase contrast images correspond to potential distributions in the case of thin samples. Since the phase contrast is sensitive to light elements, DPC-STEM could be effective observation method for polymer materials. In the present study, we have tried to visualize the three-dimensional structure of non-stained polymer alloy using DPC-STEM tomography.

Acrylonitrile-butadiene-styrene (ABS) polymer alloy that acrylonitrile-styrene (AS) phase and butadiene (B) phase are separated is used for the analysis. The sample is thinned by cryo-focused ion beam (FIB) method. An aberration corrected STEM (JEOL : JEM-ARM200F) equipped with a segmented annular all-filed detector (JEOL : SAAF Octa) was used for acquiring the DPC-STEM images. The accelerating voltage was set to $200 \mathrm{kV}$. Potential calculations were performed by a commercial program (HREM Research Inc. : qDPC).

Figure 1a shows a high angle annular dark field (HAADF)-STEM image of the ABS. Voids and inorganic filler particles such as magnesium oxide and calcium oxide which are identified by energy dispersive X-ray spectroscopy (EDX) are observed, whereas the phase-separated structure of the AS phase and the B phase is not observed. In the DPC-STEM image shown in figure 1b, round shape patterns are clearly visualized. The patterns indicate the positions of the interface between the AS phase and the B phase. Figure 1c shows the potential distribution calculated from the electrical field image at the same observation area with Figs. 1a and 1b. The contrast of the AS phases, the B phases, the filler particles, and the voids are significantly enhanced compared with the HAADF-STEM image.

In order to analyze the three-dimensional structure of the ABS polymer alloy, the electron tomography is performed based on the tilt-series of the potential maps acquired by DPC-STEM. After the DPC-STEM images are acquired over an angular range of $-72^{\circ}$ to $72^{\circ}$ in $4^{\circ}$ increments, the potential maps of each electrical field image are calculated. The tilt-series is aligned and reconstructed using filtered back projection (FBP) method. The potential map obtained at $0^{\circ}$ is shown in figure $2 \mathrm{a}$. Green rectangle 
indicates the reconstruction area. The reconstructed volume data is shown in figure $2 \mathrm{~b}$. The B phases are reconstructed as oval sphere shapes. The filler particles are included only in the AS phases, whereas the voids are included in both the AS phases and the B phases.

In summary, we have successfully observed the phase-separated structure of polymer alloy without electron staining by using DPC-STEM. Image contrast of the filler particles and the voids in the polymer alloy is also enhanced. Furthermore, the three-dimensional analysis has been demonstrated using DPCSTEM tomography. It was shown that DPC-STEM is powerful technique for visualizing original structure of non-stained polymer materials.

\section{References:}

[1] K Kato, J. Electron Microsc. 4 (1966), p. 35.

[2] N Shibata et al., Sci. Rep. 5 (2015), p. 10040.

[3] N Shibata et al., Nat. Commun. 8 (2017), p. 15631.

[4] A Ishizuka, Microscopy 66 (2017), p. 397.
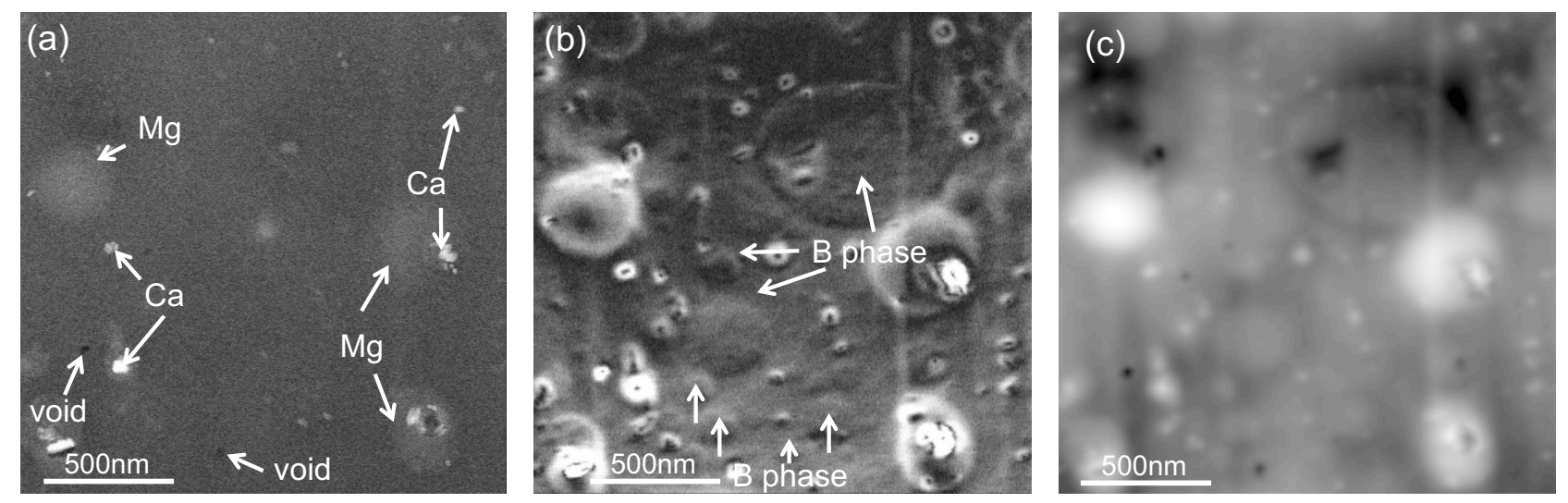

Figure 1. Observation results of the non-stained ABS polymer alloy. (a) HAADF-STEM image, (b) Electrical field acquired using DPC-STEM, and (c) potential map calculated from the electrical field.
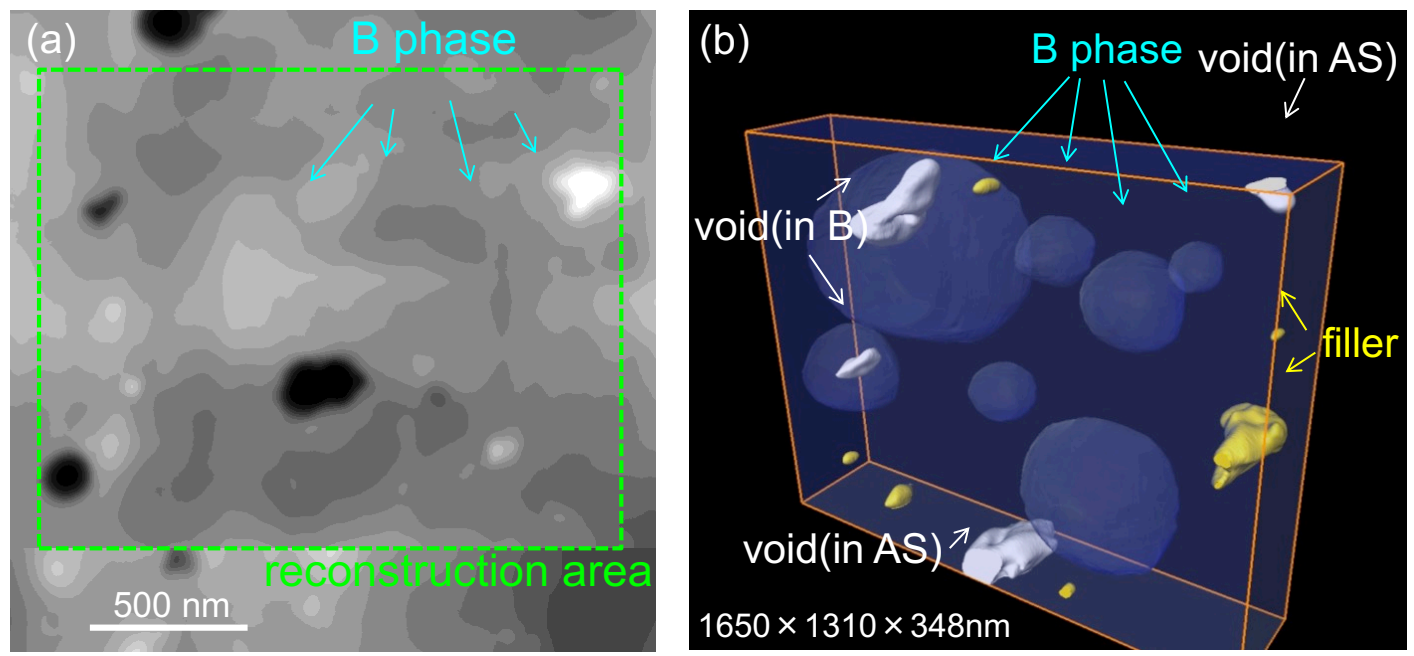

Figure 2. (a) The potential map obtained at $0^{\circ}$, (b)Three-dimensional reconstruction of the ABS polymer alloy by DPC-STEM tomography. 